\title{
Relationship of angiogenesis factor in synovial fluid to various joint diseases
}

\author{
R. A. BROWN , ${ }^{1}$ I. W. TOMLINSON, ${ }^{1} \mathrm{C}$. R. HILL, ${ }^{1} \mathrm{JACQUELINE} \mathrm{B.} \mathrm{WEISS,}$ \\ P. PHILLIPS,$^{2}$ ANDS. KUMAR ${ }^{2}$
}

From the ${ }^{1}$ Department of Rheumatology, University of Manchester Medical School, and the ${ }^{2}$ Department of Oncology, Christie Hospital, Manchester

SUMMARY A low-molecular-weight freely dialysable angiogenesis factor has been isolated from 49 synovial fluids obtained from patients with various joint diseases. An analysis of disease type and incidence of freely dialysable angiogenesis activity showed that the osteoarthrotic group had a significantly higher incidence than all the other groups $(p=0.0332)$. Angiogenesis factor has also been detected in a bound form in the retentates of fluids which gave positive results for dialysable factor. The possibility that an imbalance between carrier-bound and free factor may have a causative role in disease is discussed.

The production of soluble compound(s) capable of inducing and attracting blood vessel growth towards a tumour was first demonstrated by Folkman et al. ${ }^{1} \mathrm{~A}$ potent low-molecular-weight angiogenesis factor was later purified from crude rat Walker tumour extracts and shown to produce experimental angiogenesis in the chick chorioallantoic membrane. ${ }^{2}$ A similar factor from cultured rat Walker tumour cells has been reported to stimulate mouse kidney endothelial cells in vivo. ${ }^{3}$ Although the initial work on angiogenesis factor was based on tumour-derived material, such factors have also been extracted from other sources such as feline and bovine retina. ${ }^{45}$ We have recently demonstrated a factor, apparently identical to the low-molecular-weight angiogenesis factor, in human synovial fluid. ${ }^{6}$

It is well established that solid tumours require a rich blood supply, provided by capillary proliferation before they can grow beyond a few millimetres in diameter. ${ }^{1}$ Synovial capillary proliferation is also a feature of the early stages of certain joint diseases, including rheumatoid arthritis. Indeed in rheumatoid arthritis the articular cartilage is frequently penetrated by capillaries from the synovial pannus, the membranous, vascular granulation tissue adherent to the cartilage. Adult articular cartilage itself is known to contain inhibitors of angiogenesis and thus to resist vascularisation. In the rheumatoid joint there may be either a failure in production of such inhibitors in the

Accepted for publication 5 March 1982.

Correspondence to Dr Jacqueline B. Weiss, Rheumatic Diseases Centre, Clinical Sciences Building, Hope Hospital, Eccles Old Road, Salford M6 8HD. cartilage or a dramatic increase in angiogenesis factor from synovium, resulting in loss of control of capillary growth. In an earlier report we indicated that there was a possible association between the presence of a positive angiogenesis factor and the type of disease, but the numbers of patients studied were too small for statistical analysis. In this paper we report a study of the presence of angiogenesis factor in the synovial effusions of 49 patients with a variety of joint disorders. An association between the presence of dialysable angiogenesis factor in synovial fluid and osteoarthritis has been established.

\section{Materials and methods}

Synovial fluids were obtained at therapeutic aspiration, normally from the knee joint, from patients with a variety of rheumatological disorders. The patients were divided into 4 diagnostic groupings: (I) seropositive rheumatoid arthritis; (II) seronegative inflammatory arthritis (including ankylosing spondylitis, psoriatic arthritis, and Reiter's syndrome); (III) osteoarthrosis; (IV) other conditions (essentially, post-traumatic synovitis).

The patients with rheumatoid arthritis (group I) fulfilled the American Rheumatism Association (ARA) criteria for classical or definite rheumatoid arthritis. Diagnoses of all other patients were made on the basis of clinical, radiological, and serological assessment. Fluids were centrifuged at $700 \mathrm{~g}$ for 5 minutes to remove any cells and the supernatant stored at $-20^{\circ} \mathrm{C}$ until extraction. 
Extraction of synovial angiogenesis factor by dialysis (method 1). Angiogenesis factor was subsequently extracted from the cell-free supernatant by the method of Brown et al. ${ }^{6}$ This method detects only the freely dialysable factor.

Extraction of synovial angiogenesis factor from nondialysable components of synovial fluids (method 2). Synovial fluid $(10 \mathrm{ml})$ was extracted by dialysis (method 1) and the dialysate was positive on the chick choriollantoic membrane assay (CAM). The retentate was then made up to $15 \%$ with trichloroacetic acid (TCA) by careful addition of a $100 \%$ TCA solution to the stirred fluid. The resultant white precipitate was spun down to give a pellet which was extracted with $0.2 \mathrm{M}$ acetic acid for 20 minutes at room temperature with gentle shaking.

Low-speed centrifugation ( $1500 \mathrm{~g}$ for $30 \mathrm{~min}$ ) of this material gave a milky supernatant, which was filtered through Whatman number 1 paper and dialysed against 5 changes of 10 volumes of $50 \mathrm{mM}$ tris $/ \mathrm{HCl}$ buffer, $\mathrm{pH} 7 \cdot 3$, at $4^{\circ} \mathrm{C}$. The retenate, at this stage a clear solution, was applied to a diethylaminoethyl (DEAE) 52 cellulose ion exchange column (Whatman, Maidstone, Kent), and bound material was eluted with a convex gradient of sodium chloride from $0-0.3 \mathrm{M}$, in $50 \mathrm{mM}$ tris $/ \mathrm{HCl}, \mathrm{pH} 7 \cdot 3$, at $4^{\circ} \mathrm{C}^{2}$ Pooled eluates from the DEAE cellulose column were further purified by affinity chromatography using antibody to crude tumour angiogenesis factor ${ }^{267}$ bound to $\mathrm{CNBr}$ activated Sepharose 4B matrix (Pharmacia, Uppsala, Sweden). Nonspecifically bound components were removed by washing the column with $0.5 \mathrm{M} \mathrm{NaCl}$ in $50 \mathrm{mM}$ tris $/ \mathrm{HCl} \mathrm{pH}$ $7 \cdot 3$ (running buffer) until a flat baseline was obtained (monitored at $280 \mathrm{~nm}$ ). Bound angiogenesis factor was then eluted with $50 \mathrm{mM}$ ammonium acetate $\mathrm{pH}$ $3 \cdot 7$.

Both the dialysable material prepared by method 1 and the material from the retentate by method 2 were prepared for assay on the chorioallantoic membrane by the addition of $10 \mathrm{mg}$ of lactose to the solutions, which were then sterilised by millipore filtration and freeze dried. The lactose acted a bulking agent to facilitate handling of the trace amounts of angiogenesis factor. The CAM assay was as described previously. ${ }^{7}$ Both lactose itself and the nonvolatile components of the tris buffer were negative when tested on the CAM assay. A general scheme for the 2 methods of preparation of angiogenesis factor is given in Fig. 1. Tumour angiogenesis factor was purified from rat Walker tumour for comparison of DEAE elution profiles. ${ }^{2}$ Data were analysed by the

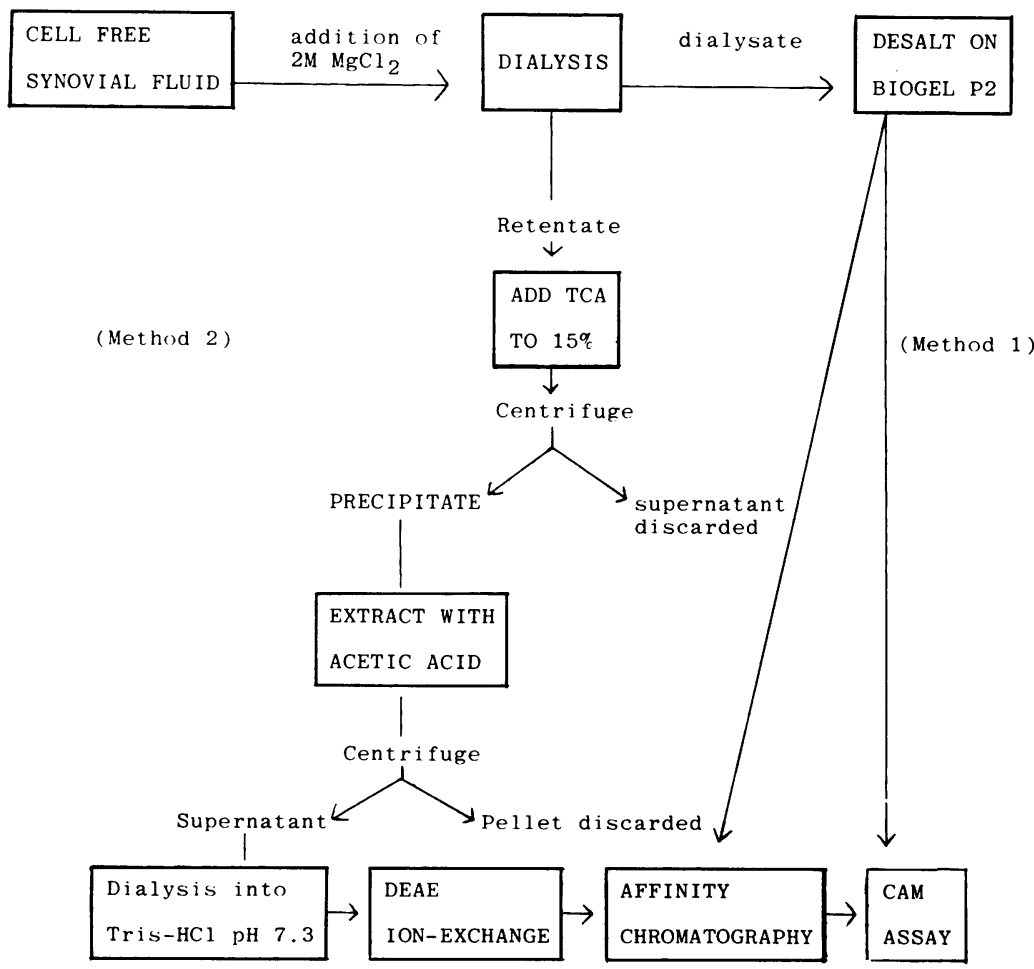

Fig. 1 Flow diagram summarising the 2 extraction methods ( 1 and 2 ) used in this study. 
Table 1 Distribution of positive CAM assays by diagnosis: patients

\begin{tabular}{|c|c|c|c|c|c|c|c|c|c|}
\hline & \multicolumn{2}{|c|}{ Group I } & \multicolumn{2}{|c|}{ Group II } & \multicolumn{2}{|c|}{ Group III } & \multirow{2}{*}{$\frac{\text { Group IV }}{\text { No. }}$} & \multicolumn{2}{|c|}{ Total } \\
\hline & No. & $\%$ of group & No. & $\%$ of group & No. & \% of group & & No. & $\%$ of total \\
\hline $\begin{array}{l}\text { CAM-positive } \\
\text { CAM-negative } \\
\text { Total }\end{array}$ & $\begin{array}{r}4 \\
19 \\
23\end{array}$ & $\begin{array}{l}17 \\
83\end{array}$ & $\begin{array}{r}6 \\
10 \\
16\end{array}$ & $\begin{array}{l}38 \\
62\end{array}$ & $\begin{array}{l}4 \\
3 \\
7\end{array}$ & $\begin{array}{l}57 \\
43\end{array}$ & $\begin{array}{l}0 \\
3 \\
3\end{array}$ & $\begin{array}{l}14 \\
35 \\
49\end{array}$ & $\begin{array}{l}29 \\
71\end{array}$ \\
\hline
\end{tabular}

$\mathrm{p}=\mathbf{0} \cdot \mathbf{1 1 0}$.

Table 2 Distribution of positive CAM assays by diagnosis: synovial fluids

\begin{tabular}{|c|c|c|c|c|c|c|c|c|c|}
\hline & \multicolumn{2}{|c|}{ Group I } & \multicolumn{2}{|c|}{ Group II } & \multicolumn{2}{|c|}{ Group III } & \multirow{2}{*}{$\frac{\text { Group IV }}{\text { No. }}$} & \multicolumn{2}{|c|}{ Total } \\
\hline & No. & $\%$ of group & No. & $\%$ of group & No. & $\%$ of group & & No. & $\%$ of total \\
\hline CAM-positive & 6 & 20 & 9 & 38 & 6 & 67 & 0 & 21 & 32 \\
\hline CAM-negative & 24 & 80 & 15 & 62 & 3 & 33 & 3 & 45 & 68 \\
\hline Total & 30 & & 24 & & 9 & & 3 & 66 & \\
\hline
\end{tabular}

$\mathrm{p}=0.0332$.

Statistical Package for Social Sciences on the University of Manchester Regional Computer Centre's ICL 1906a/CDC 7600 computer. Comparisons were made by the nonparametric tests Kruskal-Wallace $\mathrm{Y}$ analysis of variants and Mann-Whitney $U$ test.

\section{Results}

Sixty-six aspirates were extracted for angiogenesis factor (by method 1) and tested on the CAM assay. A total of 49 patients were involved in the survey (Table 1), though in some cases synovial fluid was aspirated from different joints or on different occasions from the same patient (Table 2). $32 \%$ of all the aspirates tested contained angiogenesis factor.

In terms of patients $29 \%$ of the complete group of 49 individuals produced synovial fluid which was positive by the CAM assay. Twelve of the patients had joints aspirated on more than one occasion (Table 3). This complicated the analysis, since in some cases repeat aspirates from the same individual gave different results. Where comparisons have been made between patients, they have been assigned arbitrarily to angiogenesis positive or negative groups on the basis of the first aspirate to be analysed.

In general the male patients tended to be younger than females $(p=0.005)$ and more fluid was aspirated from males $(p=0 \cdot 014)$. Disease duration was similar in both sexes. The female-to-male ratio of patients tested was $1 \cdot 28: 1$, but sex of the individual had no significant bearing on the outcome of the test.

Out of the 23 patients in group I (rheumatoid) 4 gave positive CAM assays. These 4 individuals produced larger volumes of fluid $(p=0.042)$ and had lower blood white cell counts $(p=0 \cdot 041)$ than the CAM-negative patients. The drug regimen of patients within this group had no detectable effect on the appearance of angiogenesis activity.

Six of the 16 patients from group II (seronegative arthritis) gave synovial fluids containing angiogenesis factor. The tendency for males to predominate in this

Table 3 Multiple extractions: patients who at different times gave more than one fluid for extraction. Each patient's aspirates are arranged in the order that they were taken

\begin{tabular}{|c|c|c|c|}
\hline Patient & $\begin{array}{l}\text { Aspirate } \\
\text { number }\end{array}$ & $\begin{array}{c}\text { Disease/ } \\
\text { category }\end{array}$ & $\begin{array}{l}C A M \\
\text { assay }\end{array}$ \\
\hline \multirow[t]{3}{*}{1} & 1 & AS/II & - \\
\hline & 2 & AS/II & + \\
\hline & 3 & AS/II & + \\
\hline \multirow[t]{4}{*}{2} & 1 & Seronegative & + \\
\hline & 2 & arthritis/II & - \\
\hline & 3 & arthritis/II & + \\
\hline & 4 & arthritis/II & - \\
\hline \multirow[t]{2}{*}{3} & 1 & Polyarthritis/II & - \\
\hline & 2 & Polyarthritis/II & - \\
\hline \multirow[t]{2}{*}{4} & 1 & Reiter's/II & weak + \\
\hline & 2 & Reiter's/II & - \\
\hline \multirow[t]{2}{*}{5} & 1 & Reiter's/II & - \\
\hline & 2 & Reiter's/II & - \\
\hline \multirow[t]{2}{*}{6} & 1 & OA/III & + \\
\hline & 2 & OA/III & + \\
\hline \multirow[t]{2}{*}{7} & 1 & OA/III & + \\
\hline & 2 & OA/III & weak + \\
\hline \multirow[t]{2}{*}{8} & 1 & RA/I/(OA III) & + \\
\hline & 2 & $\mathrm{RA} / \mathrm{I} /(\mathrm{OA}$ III) & weak + \\
\hline \multirow[t]{3}{*}{9} & 1 & $\mathbf{R A} / \mathbf{I}$ & - \\
\hline & 2 & $\mathrm{RA} / \mathrm{I}$ & - \\
\hline & 3 & RA/I & + \\
\hline \multirow[t]{2}{*}{10} & 1 & RA/I & - \\
\hline & 2 & RA/I & - \\
\hline \multirow[t]{2}{*}{11} & 1 & RA/I & - \\
\hline & 2 & $\mathbf{R A} / \mathbf{I}$ & - \\
\hline \multirow[t]{2}{*}{12} & 1 & RA/I & - \\
\hline & 2 & RA/I & - \\
\hline
\end{tabular}


CAM-positive group was not significant. Both of the patients with classical ankylosing spondylitis were positive, but the 2 cases of Reiter's syndrome were negative (retests from the same patients gave only one weak positive result).

More than half of the patients -4 out of 7 -in group III (osteoarthrosis) were positive for angiogenesis factor, easily the highest incidence in the survey. When fluids from this group were tested on another occasion (Table 3 ) they were again positive. In contrast group IV contained no patients who gave positive aspirates by CAM assay.

Comparison of the 4 groups (I-IV) suggested that differences existed in age $(p=0.0001)$, duration of disease $(p=0 \cdot 042)$, and ESR $(p=0 \cdot 0017)$. Members of group II were younger than those in group I (RA; $p=0.0002)$ and group III (OA; $p=0.005)$. Individuals from group II also had a shorter disease duration than those of group I $(p=0.02)$. In general the patients with positive CAM assays had given greater volumes of synovial fluid at aspiration than CAM-negative patients $(p=0 \cdot 024)$. However, subdividing the indiviuals still further suggests that only females and group I (RA) patients showed this trait to a significant degree $(p=0.024$ and 0.043 respectively).

The difference in incidence of positive assays in the fluids between the diagnostic groups was significant $(p=0.033)$ and remained so even when the miscellaneous group (group IV) was excluded.

\section{MULTIPLE EXTRACTIONS}

Twelve patients in the group had their joints aspirated and fluids tested on more than one occasion. In some cases as many as 4 results were available for a single patient. Table 3 shows the results of patients who had had multiple extractions. Seven of these 12 patients were 'positive' for angiogenesis factor at
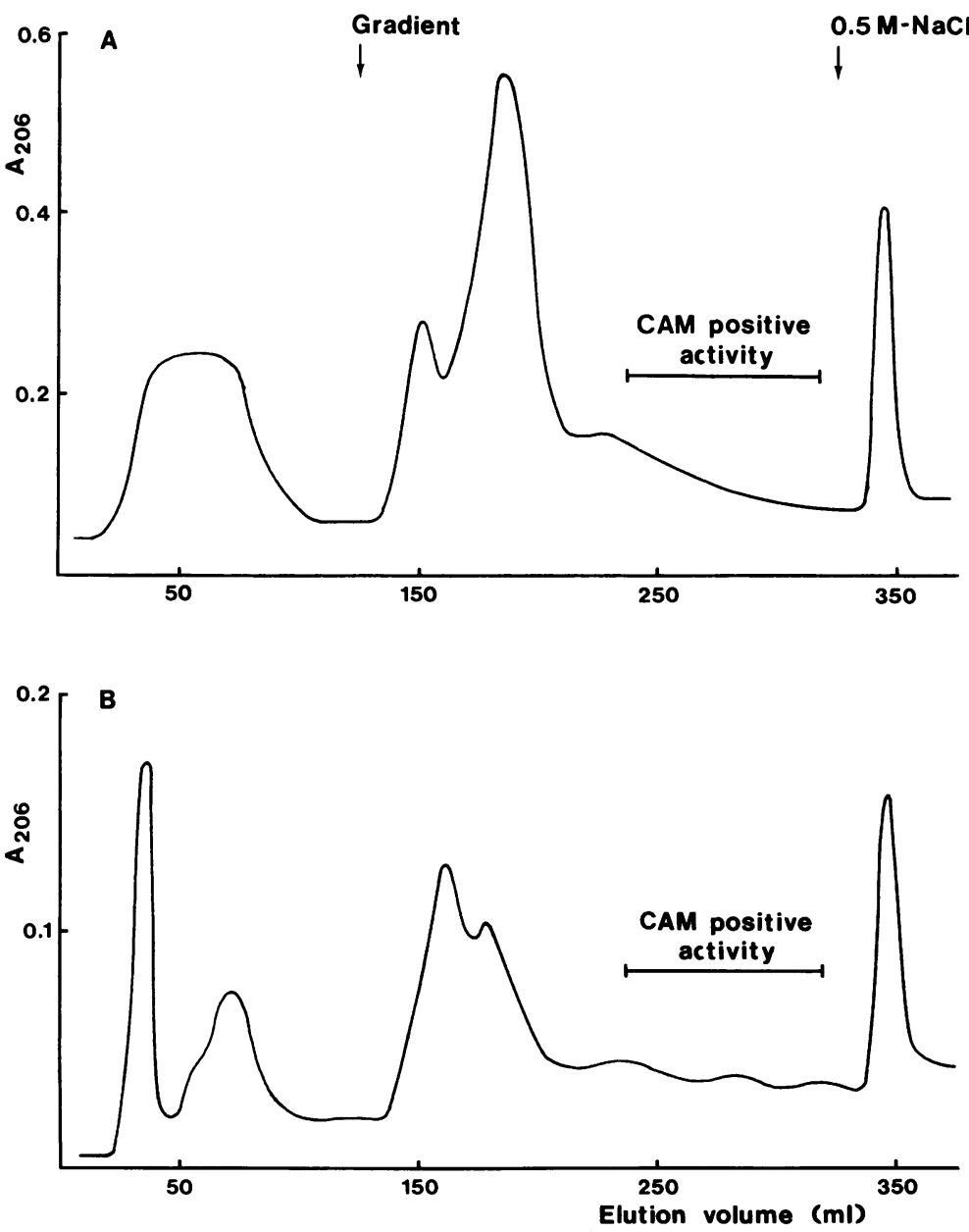

Fig. 2 DEAE cellulose chromatography of synovial extract (A) and Walker 256 tumour extract (B). A column $(10 \times 1 \mathrm{~cm})$ of DEAE 52 cellulose (Whatman) was equilibrated with $50 \mathrm{mM}$ tris/ $\mathrm{HCl}$ buffer pH 7.3 at $4^{\circ} \mathrm{C}$. Unbound material was eluted with the same buffer at a flow rate of $50 \mathrm{ml} / \mathrm{h}$. The column effluent was monitored at $206 \mathrm{~nm}$ (Uvicord S, LKB, Sweden). Bound material was eluted with a convex salt gradient $(0-0.3 \mathrm{M} \mathrm{NaCl})$ prepared in a constant level mixing device from $50 \mathrm{mM}$ tris/ $\mathrm{HCl} \mathrm{pH} \mathrm{7.3} \mathrm{(65} \mathrm{ml})$ and $50 \mathrm{mM}$ tris $/ \mathrm{HCl}$ containing $0 \cdot 3 \mathrm{M}-\mathrm{NaCl}(100 \mathrm{ml})$. The column was finally washed with $50 \mathrm{mM}$ tris/ $\mathrm{HCl}$ containing $0.5 \mathrm{M} \mathrm{NaCl}$. 
some time, 5 patients on more than one occasion. Twelve fluids ( $41 \%$ ) from this selected group gave an angiogenic response, considerably more than the overall positive rate for the whole survey $(32 \%)$.

The distribution of CAM-positive fluids within the disease categories was similar to that in the main study. The most consistently positive aspirates were obtained from patients with primary or secondary osteoarthrosis. Seropositive rheumatoid fluids were occasionally CAM-positive (e.g. patient 9 , positive once in 3 tests).

Many of these repeat tests gave conflicting results, for example, patient 2 had 2 positive and 2 negative aspirates and patient 1 had 2 positive and 1 negative aspirate. This tendency is most pronounced in patients with more than 2 results, namely, patients 1 , 2,9 , and 12 . Only the last gave 3 consistent results, suggesting that there is a further, unidentified factor operating in the appearance of angiogenesis factor in synovial fluid.

ALTERNATIVE METHOD FOR THE EXTRACTION OF ANGIOGENESIS FACTOR

The routine extraction and assay technique developed during this study tests only for initially dialysable angiogenesis factor. It has proved difficult to test directly the whole synovial aspirate or the retentate after dialysis, and so we have developed an extraction method based on that used for crude tumour angiogenesis factor. ${ }^{2}$ Extraction of the retentate after dialysis is summarised in Fig. 1 as 'method 2'. Method 2 serves partly as a comparison with tumour-derived angiogenesis factor and partly as a measure of the dissociation of angiogenesis factor from its carrier.

The starting material was the retentate left after a standard extraction (method 1) from a fluid which gave a positive result by the conventional dialysis method. The subsequent treatment involved extraction from a precipitate and a second dialysis stage before DEAE ion exchange and affinity chromatography. Clearly the nature of this extraction should eliminate any unbound low-molecular-weight angiogenesis factor before the chromatographic steps. DEAE elution profiles in Fig. 2 show the synovial extract and a comparable separation of crude angiogenesis factor from rat Walker tumour. CAMpositive material was obtained from the same area of elution profile in both cases, activity eluting in an anionic position at the end of the gradient. After affinity chromotography the CAM-positive material became freely dialysable, indicating that it had dissociated from a carrier molecule during this process.

\section{Discussion}

A total of 66 synovial fluids from 49 separate patients were tested in this survey. One-third of the fluids tested were positive on the CAM assay. A similar proportion of positive assays was found when the results were presented in terms of individual patients. However, of the 12 patients who had fluid aspirated on more than one occasion half gave positive results. This could suggest that the more often a synovial fluid is aspirated (that is, in the case of chronic synovial effusions) the greater the likelihood that this angiogenesis factor will be detected. Alternatively it could be argued that the presence of angiogenesis factor in the joint could itself lead to the excessive production of synovial fluid. However, it must be said that the results presented may be modified by inadequacies of the extraction procedure, which depend on the concentration of carrier molecules in the aspirate. Since the angiogenesis factor is a low-molecularweight material, it is possible that in the absence of carrier it might diffuse rapidly out of the joint space.

We demonstrated the presence of both freely dialysable and bound angiogenesis factor in a synovial fluid. The bound material also became freely dialysable after it had passed through an affinity column containing an antibody to a crude tumour angiogenesis factor. Possibly the proportion of bound to unbound material influences the number of positive results obtained by the dialysis method. Whether or not the amount of carrier relative to free angiogenesis factor is related to the disease process is a matter for further study, and this is under way.

Dialysable angiogenesis factor was obtained more often in the case of affected males in inflammatory arthritis, but there was no difference in the sex distribution for the small osteoarthrotic group. Among the disease groups studied the incidence of angiogenesis activity was lowest in patients with rheumatoid arthritis. This was unexpected, particularly in view of the marked synovial proliferation with hyperaemia which is characteristic of the pathology of rheumatoid arthritis. In contrast the proportion of extractable angiogenesis factor in the osteoarthrotic group was very high. This would imply that the production of angiogenesis activity is not specifically attributable to inflammatory cells, since the osteoarthrotic fluids are known to contain very few such cells, and in those fluids which we examined ourselves there were too few inflammatory cells to be counted.

In a study of cell populations in synovial fluids, Naid showed that there were relatively large numbers of chondrocytes and synoviocytes in fluids from patients with osteoarthroses but found no evidence for cartilage cells in rheumatoid effusions. The idea that cartilage fragments in the synovial fluid produce angiogenesis factor is tempting. It is known, for example, that chondrocytes can alter their collagen 
phenotype in different environments ${ }^{9-11}$ and can become metabolically more active at the surface of either OA or experimentally injured articular cartilage. ${ }^{12}$ Perhaps these cells, removed from their normal matrix, begin to secrete an angiogenesis factor. The presence of such a factor would presumably lead to the formation of an effusion and vascular changes in the synovial membrane.

On the other hand it could be argued that rheumatoid patients represent a different case from those with osteoarthrosis in that no extra angiogenesis factor is produced. Rather the cartilage no longer produces sufficient inhibitor of blood vessel invasion, ${ }^{13}$ leading to an imbalance between the stimulation and inhibition of angiogenesis. For whatever reason, however, it is clear that no simple relationship exists between capillary proliferation in the synovial membrane and the presence of extractable angiogenesis factor. Though the inadequacies of the extraction method may influence this finding, they would be the same for all fluids tested. The low incidence of positive results in the RA group could reflect clearance due to the vascular perfusion of the joint. Despite evidence for the presence of inflammatory and vascular changes in some forms of osteoarthrosis ${ }^{14-16}$ these features are far more pronounced in rheumatoid synovial membranes. ${ }^{17}{ }^{18}$ Interestingly, it has been suggested ${ }^{17}$ that the vascular changes in the 2 diseases differ more in intensity and distribution than in form.

It cannot of course be ruled out that the patients positive for angiogenesis factor in the rheumatoid group may represent a subpopulation. These patients may have a different cell distribution pattern, perhaps with a higher number of synoviocytes, than is usual, and we are currently investigating this point.

It may be worth pointing out that early work on the ultrastructure of synovial, membranes in rheumatoid arthritis has shown some cases where mast cells were evident. ${ }^{18}$ These cells have been described in all areas where angiogenesis is taking place, including the chick chorioallantoic membrane itself (R. A. Fraser and J. G. Simpson, personal communication). Since these cells secrete heparin, which has been shown to be synergistic with endothelial-cell-stimulating angiogenesis factor (ESAF), although having no angiogenic activity itself ${ }^{19}$ their presence may be significant in this respect. A recent study of synovial ultrastructure has shown that mast cells have been observed at the pannus-cartilage junction in some specimens of rheumatoid synovial (M. Bromley and D. Woolley, personal communication). In view of our own finding of synovial fluids from rheumatoid patients being positive for angiogenesis activity in only a proportion of cases, this may be significant.

It is important in interpreting the results reported in this paper to clarify the term 'weak positive' as it relates to the CAM assay response (see Table 1). From dilution studies we have evidence to suggest that the weak positive response indicates low concentrations of angiogenesis factor. The results of the survey are set out on the assumption that these samples were positive for angiogenesis factor.

In this survey we have established that a lowmolecular-weight angiogenesis factor can be extracted from synovial fluids produced in a variety of joint diseases. A part of the factor is freely dialysable after treatment of the synovial fluid with $2 \mathrm{M} \mathrm{MgCl}_{2}$, the remainder being carrier bound. The role of this factor in the pathogenesis of joint diseases had yet to be established.

We thank Mrs Shirley Johnson for expert technical assistance and Dr V. Hillier for her help with the statistical analyses. This work was supported in part by a grant from Arthritis and Rheumatism Council for Research.

\section{References}

1 Folkman J, Merler M, Abernathy C, Williams C. Isolation of a tumour factor responsible for angiogenesis. J Exp Med 1971; 133: 275-88.

2 Weiss J B, Brown R A, Kumar S, Phillips P. Tumour angiogenesis factor: a potent low molecular weight compound. BrJ Cancer 1979; 40: 493-6.

3 McAuslan B R, Hoffman H. Endothelial stimulating factor from Walker carcinoma cells. Exp Cell Res 1979; 199: 181-90.

4 Kissun R D, Hill C R, Gamer A, Phillips P, Kumar S, Wiess J B. A low molecular weight angiogenic factor in cat retina. $\mathrm{Br} \mathrm{J}$ Ophthalmol 1982; 66: 165-9.

5 D'Amore P A, Glaser B M, Brunson S K, Fenselau A H. Angiogenic activity from bovine retina: partial purification and characterization. Proc Natl Acad Sci USA 1981; 78: 3068-72.

6 Brown R A, Weiss J B, Tomlinson I W, Phillips P, Kumar S. Angiogenic factor from synovial fluids resembling that from tumours. Lancet 1980; 1: 682-5.

7 Phillips $P$, Kumar S. Tumour angiogenesis factor and its neutralisation by xenogeneic antiserum. Int J Cancer 1979; 23: 82-8.

8 Naid Z M, Cytology of synovial fluids. Acta Cytol (Baltimore) 1973; 17: 299-309.

9 Norby D P, Malemud C J, Sokoloff L. Differences in the collagen types synthesised by lapine articular cartilage chondrocytes in spinner and monolayer culture. Arthritis Rheum 1977; 20: 709-16.

10 Von der Mark K, Gauss V, Von der Mark H, Muller P. Relationship between cell shape and type of collagen synthesis as chondrocytes loose their cartilage phenotype in culture. Nature 1977; 267: 531-2.

11 Benja P D, Padilla S R, Nimni M E. The progeny of rabbit articular chondrocytes synthesise collagen types I and II and type I trimer but not type II. Verification by cyanogen bromide peptide analysis. Biochemistry 1977; 16: 865-72.

12 Ball J, Sharp J. Osteoarthrosis. In: Scott J T, ed. Copeman's Textbook of the Rheumatoid Diseases. London and Edinburgh: Churchill Livingstone, 1978: 595-644.

13 Brem H, Folkman J. Inhibition of tumour angiogenesis mediated by cartilage. J Exp Med 1975; 141: 427-39.

14 Roy S. Ultrastructure of synovial membrane in osteoarthritis. Ann Rheum Dis 1967; 26: 517-27.

15 Peter J B, Pearson G M, Marmor L. Erosive osteoarthritis of the hands. Arthritis Rheum 1966; 9: 365-88. 
16 Huskisson E C, Dieppe P A, Tucker A K, Cannell L B. Another look at osteoarthritis. Ann Rheum Dis 1979; 38: 423-8.

17 Goldie I. The synovial microvasculature derangement in rheumatoid arthritis and osteoarthritis. Acta Orhtop Scand 1970; 40: 751-66.
18 Norton W L, Ziff M. Electron microscopic observations on the rheumatoid synovial membrane. Arthritis Rheum 1966; 9: 589-610.

19 Azizkhan R G, Azizkhan J C, Zetter B R, Folkman J. Mast cell heparin stimulates migration of capillary endothelial cells in vitro. J Exp Med 1980; 152: 931-44. 